\title{
THE ACTIVE AREA IN COURSE OF EXCITATION CONDUCTION (OBSERVATIONS ON LILLIE'S NERVE MODEL)
}

\author{
By KAZUMI YAMAGTWA \\ Department of Physiology, Tokyo Medico-Dental College, Tokyo
}

\section{INTRODUCTION}

We have hitherto had two kinds of core-conductor models for the nerve, namely, one of Hermann's and the other of Lillie's type. They are similar in structure, both being a metal wire (the core) immersed in a certain electrolyte solution (the sheath fluid). The Lillie's model, an iron core immersed in strong nitric acid, has, however, a greater advantage in the sense that it can be "stimulated" or "activated" and, owing to the "activation current", which flows between the activated and the resting regions just like the action current in the nerye, the neighbouring points can be activated one after another just as suggested in Hermann's core conductor theory of nerve conduction. We have thus the phenomenon of "conduction" in Lillie's model, and as the process is accompanied by a distinct colour change, we can follow the conduction of the "activation wave" quite easily with our naked eyes. This model was invented by R.S. Lillie in 1916, and ever since a great many interesting observations have been made on it by the inventer himself. The present author, having been engaged in the studies of Hermann's model for several years, took Lillie's one, to examine a few ideas of his own with regard to nerve conduction. Of course a model is a model after all, and there is no ground to deduce anything physiological from the results obtained; nevertheless, it is not always unuseful, because the studies can be done on it in such ideal conditions as they are not realisable in living tissues, and the results obtained often give important hints to new facts or theories.

The object of the present work is to examine the "length" of the' activation wave and the variations which it suffers in course of conduction along a core under uniform or non-uniform conditions.

\section{PRELIMINARY TESTS ANd REMARKS}

1. On the core

The wire to be used as the core should be polished well, first with a piece of the finest sand paper, then with some cloth, and then washed with water, wiped with clean cloth to be immersed in a bath of strong nitric acid to make it "passive". If polished roughly or not uniformly, the activation wave cannot be conducted with any regularity. Another important point to be remarked is that the time of immersion necessary for a sufficient passivation is different from core to core when made from different samples, sometimes even when made from one and the same sample. The reason is not quite clear, but probably it is due to the difference in the constituents and in the way of 
polishing. At any rate, it,varies from several minutes to forty or fifty minutes. The conduction velocity, too, differs so much from core to core, that some studies, for example comparative studies on the conduction velocities in thin and thick cores, are not easy to realise, even though they appear very simple.

2. On the nitric acid

As is well known, an iron wire dipped in strong nitric acid (specific gravity $>1.3$ ) will be covered with a protective membrane of $\mathrm{Fe}_{2} \mathrm{O}_{3}$ over the surface, which prevents the chemical reaction between the iron and the acid. This state is called the passive or the inactive. The iron without this membrane is said to be in the active state. Now, between the active and the passive irons, there exists a potential difference of about $0.7-0.8 \mathrm{~V}$, the passive iron being positive. If, then, the passive membrane is partly destroyed and the active iron is exposed there in some way or another, there flows a local electric current (the activation current) in the direction active iron $\rightarrow$ acid $\rightarrow$ passive iron. The passive part which receives this current with sufficient intensity becomes active and then acts as the source of a new local current, and so on. This is the principle of the conduction of the so-called activation wave, which closely resembles that of nerve

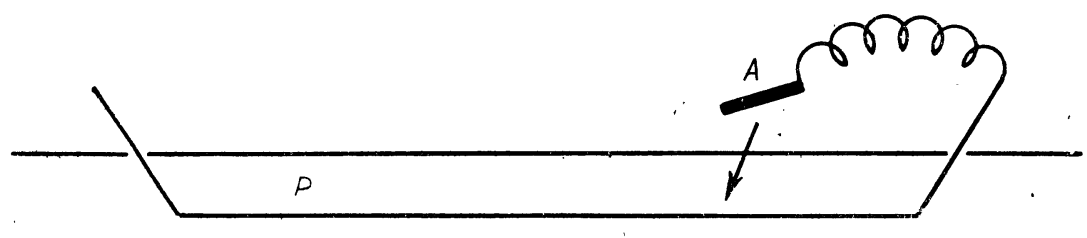

Fig. 1

conduction. This process is accompanied by a distinct colour change, a temporary blackening of the surface, so we have practically a progression of a black wave. If, now, the acid is sufficiently strong (specific gravity $>1.35$ ), the wire is repassivated automatically, and the colour turns back to white again. Here we have a progression of a white wave, which follows immediately after the black one.

The volume of the acid, the bathing fluid, plays a rôle in determining the conduction velocity, that is to say, the velocity is larger in a larger quantity of the fluid (Lillie ${ }^{1)}$ ). If we wish, therefore, to examine the variation of the velocity in cource of conduction, we ought to have 1) a uniform distribution of the fluid along the core to avoid the local irregularities, and 2) the fluid quantity as small as possible, to make the velocity small and the observations easier. But as the repassivation gets difficult in very little acid, 3) the fluid must not be too small in quantity.

3. On the methods of activation or stimulation

To the three methods given by Lillie, that is, the mechanical, the electrical and the zinc-metal touch, the author should like to add a new one by using a piece of active iron (Fig. 1). In the figure, $\mathrm{P}$ is the passive core, and $\mathrm{A}$ is a piece of active iron connected with $\mathbf{P}$ in the air with a soft copper wire. For stimulation, $A$ is dropped 
suddenly and quickly into the nitric acid somewhere near the core, and that is all. It was noticed that the length of $\mathrm{A}$, its direction relative to $\mathrm{P}$, etc. are significant with regards to the effect. This procedure is something like that in the case of "twich without metal" in nerve-muscle preparations.

4. The activation wave of limited length and its significance in the present work

We have learnt that if a passive core is stimulated in strong nitric acid, we have first the activation wave (black) going on, and then the repassivation wave (white) running after that. If, therefore, the velocity is relatively slow and the repassivation (the recovery) is relatively quick, there is a possibility of having a black activation wave of a certain clearly limited length. Let it be called the "length" of the wave or simply the "wave-length". Lillie himself observed this in his later experiments (Lillie2), 3)), but he did not go much further on it, although, standing on it, he put forward a very useful idea as to the conduction velocity. The author, however, has a 'special interest in the "length" itself, because it plays a very important rôle in his theory of nerve conduction (Yamagiwa $\left.{ }^{7}\right)$ ), and that is why he planned to examine it precisely in the present work.

According to Lillie, the only condition for obtaining a wave of limited length is to stimulate the core in "strong" nitric acid. This is indeed the most important condition, but not the sufficient one. A high grade of passivity and a relatively small quantity of the acid are also indispensable. The reason is that in strong nitric acid the passivating process is always going on; therefore, if we want to make the activation wave start and run, we have to make the activation current stronger. This requirement is met by a longer time of immersion or by a larger quantity of the acid. The latter method, however, is not suitable, because an ample reserve of strong nitric acid helps the passivation but inhibits the activation. Moreover, if the wave is once started, both the wave length and the conduction velocity will become too large to be recognized exactly. Hence, the acid should not be too great in quantity; it should not be too small either, because the repassivation process becomes then difficult, as described before. The experience taught us that waves of various limited lengths $(0.5 \mathrm{~cm}-\mathrm{a}$ few $\mathrm{cm})$ can be realised in columns of acid (specific gravity $=1.36$ ) with sections smaller than $1 \mathrm{~cm}^{2}$.

In the present work, the influences of the duration of the immersion time (that is, the grade of passivity) and that of the electrotonus will be examined.

\section{METHOD}

1. The apparatus

A long columnar groove was' made in a large mass of paraffin (Fig. 2, GG). It was filled with strong nitric acid, in which a clean polished iron wire was laid as the core. The both ends of the core were bent round to be dipped in $\mathrm{Hg}$ (see the figure) for further electric connection. The numbers from 0 to 50 on one side of the groove are those of a scale inbedded in the paraffin, which serves for measuring the length and the speed of the wave. The dimension of the groove was, for example, ca $50 \mathrm{~cm}, 5 \mathrm{~mm}$ and $3 \mathrm{~mm}$ in length, breadth and depth respectively. 


\section{Stimulation}

Either the method of Zinc-metal touch or the method of electrical stimulation was adopted. In the former case, some part of one end of the core, just exposed to the air, was covered previously with a piece of cloth soaked in the nitric acid in the groove. The covered part becomes passive, too, like the other part in the groove. At the stimulation, the piece of cloth was taken off and the exposed part was touched with a piece of Zinc-metal. The wave thus evoked proceeds quite slowly until it reaches the surface of the fluid, then suddenly much faster. As to the electrical stimulation, the method shown in Fig. 3, (a) was found very simple and effective. In the diagram, $\mathrm{Pt}$ is a piece of platinum wire, which serves as the stimulating electrode through $\mathrm{C}$, a bundle of cotton thread soaked in the nitric acid and kept in touch with the core at the part just exposed to the air. The necessary intensity of the stimulating current in such

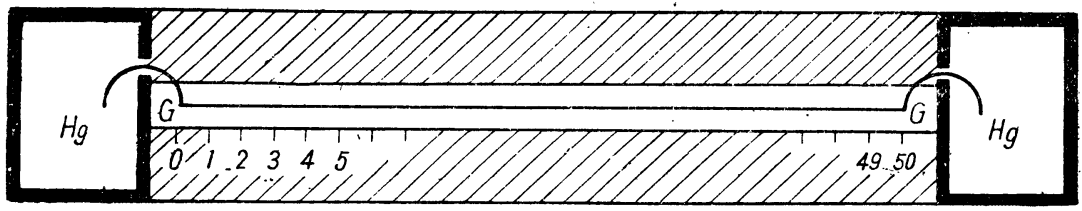

Fig. 2

(a)

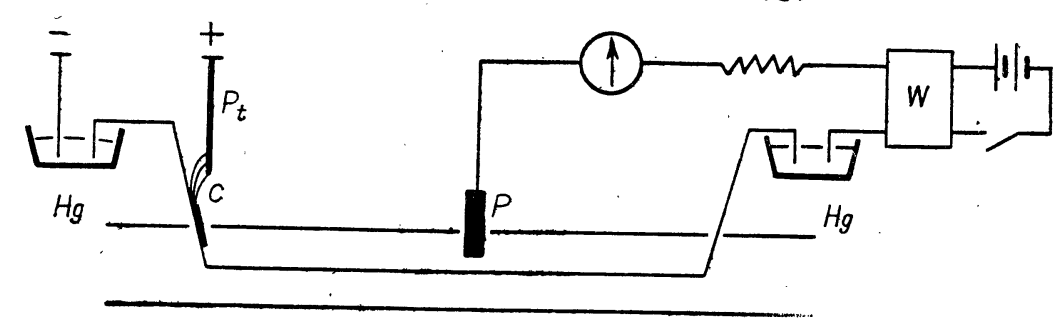

Fig. 3

(b)

a condition was found to be approximately $3-4 \mathrm{~m}$. A. at the duration of about $40 \mathrm{~m}$. sec. (N.B. In the stimulating circuit there flows a current due to the potential difference between $\mathrm{Pt}$ and $\mathrm{Fe}$ in the direction opposite to that of the stimulating. The intensity depends on the grade of passivity of the iron. This current was not compensated, because it was comparatively weak ((about $0.3 \mathrm{~m}$. A. at the strongest)) and, moreover, it served as a convenient indicater of the grade of passivity).

\section{Observation}

For exact measurements of the length or the speed of the wave, the method of cinematography should be adopted. But as we had not the apparatus, we had to be content with the observations with our naked eyes and with a stop-watch. The results obtained cannot be quantitatively said to be exact, but, we believe after repetitions of observations, they are correct qualitatively. 


\section{RESULTS}

1. The relations of the grade of passivity to the length of the wave, its conduction velocity and the variations of those in course of conduction.

The grade of passivity is a function of the time of immersion of the core in the acid. It depends, therefore, practically on the time interval between two successive activations. Now, the relation between this and the length of the wave etc. actually obtained was essentially the same in every core. It can be stated as follows:

1) A long or short interval makes the wave length large or small, and with a large or small wave length the conduction velocity becomes large or small. In each case the wave is conducted with a constant wave length, large or small.

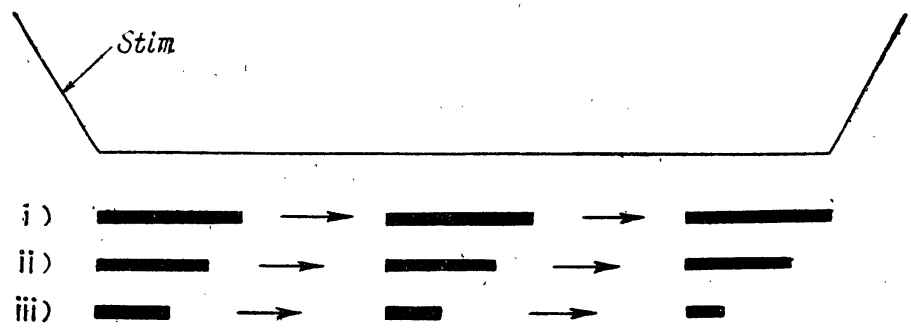

Fig. 4

Table 1

\begin{tabular}{|c|c|c|c|c|}
\hline $\begin{array}{l}\text { Moment of } \\
\text { stimulation }\end{array}$ & Wave length & Distance travelled & Temp. & Remarks \\
\hline $3^{\text {h }} 0^{\prime 1}$ ) & ca $2 \mathrm{~cm}$ (const.) & The whole length & $15.4^{\circ} \mathrm{C}$ & 1) The previous \\
\hline $20^{\prime}$ & " & " & & $\begin{array}{l}\text { stim. was given } \\
25^{\prime} \text { before. }\end{array}$ \\
\hline $35^{\prime}$ & $" \prime$ & $" \prime$ & & \\
\hline $45^{\prime}$ & $\prime \prime$ & $" \prime$ & & \\
\hline $50^{\prime}$ & ca $1.5 \mathrm{~cm}$ (const.) & $"$. & . & \\
\hline $53^{\prime}$ & ca $1 \mathrm{~cm}$ (const.) & $" \prime$ & & \\
\hline $55^{\prime}$ & ca 6-7 mm (const.) & " & & \\
\hline $57^{\prime}$ & $\|$ & $\prime \prime$ & & \\
\hline $58^{\prime}$ & ca $5 \mathrm{~mm}$ (decremental) & $8.7 \mathrm{~cm}$ & . & \\
\hline $59^{\prime}$ & $\prime \prime$ & $7.7 \mathrm{~cm}$ & & \\
\hline $59 / 30^{\prime \prime}$ & $\prime \prime$ & $5 \mathrm{~cm}$ & & \\
\hline
\end{tabular}

Note 1. We find similar descriptions on decremental conduction in some of Lillie's reports (Lillie, 5)). But they are concerned with the velocity of the wave and not with the wave length. The waves of limited lengths were found much later, in 1929 (Lillie'))

Note 2. The duration of the refractory period is different in different cores. In some core, it was just 1 or 2 min., while in some others longer than 20 or $30 \mathrm{~min}$. It was usually almost constant in each core, but sometimes it fluctuated so much that we could not carry out the experiment. In models, in which the refractory period was very short (the shortest found was $\left.1^{\prime} 15^{\prime \prime}\right)$, it was very difficult to catch the stage of "decrement", and we were apt to think as if the model followed the "all-or-none" principle as far as the wave length or the conduction velocity were concerned. 
2) In the case the interval is smaller than a certain limit, both the wave length and the cordduction velocity suffer decrements in course of conduction, until finally the wave stops to proceed somewhere on the way.

3) If the interval is too short, the activation wave does not start at all, that is, there exists a certain refractory period.

These results are shown schematically in Fig. 4, where the interval is large in i), a little smaller in ii) and much smaller in iii): The wave lengths are shown to be large and constant, smaller and constant and much smaller and decremental in i), ii) and iii) respectively. Table 1 is an example of the protocol records.

2. The influences of the electrotonus upon the length and velocity of the wave.

Lillie reported a very interesting observation with regards to the decremental conduction (Lillie $\left.{ }^{5}\right)$ ). He kept a piece of platinum foil in touch with the core in strong nitric acid. He then stimulated a point far apart from there. The activation wave came along as usual, but the velocity showed a clear decrement as the wave approached the point of contact. The wave stopped there finally. If not, the wave showed, after it had

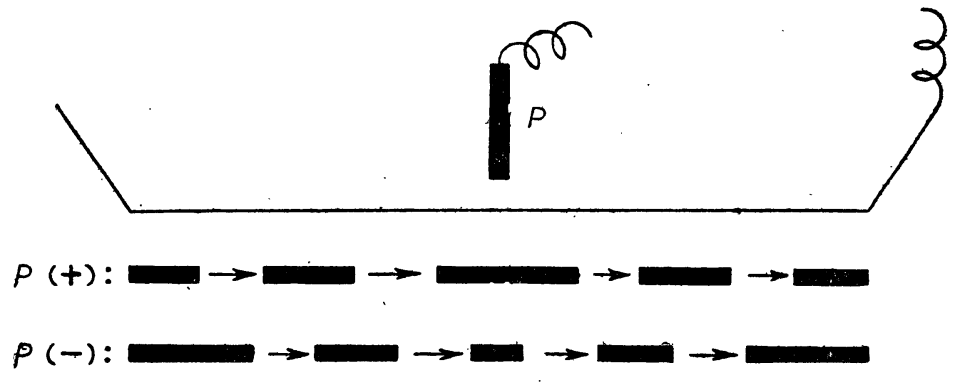

Fig. 5

passed the point, an increment in the velocity to get back gradually to the original value. This shows clearly that the catelectrotonus makes the velocity-smaller or the wave to stop, for, in the neighbourhood of the point of contact, there flows a local current in the direction iron $\rightarrow$ acid $\rightarrow$ platinum owing to the potential difference between the two metals. That is to say, the core is under the influence of the catelectrotonus (we should recall here that the activation of the core takes place under the anode, while the physiological excitation under the cathode). What will happen then, in general, in a core under the influences of cat- or anelectrotonus?

Fig. 3 (b) is the connection diagram for observing this. The polarising electrode $P$ (platinum) was set vertically as shown in the figure or parallel to the core along a few centimetres. The effect appeared clearer in the latter case. If we employ a pair of polarising electrodes instead of one, we are able to observe the effects of both kinds of electrotonus at the same time, but it then needs a much higher tension for polarising, because the shunting power of the acid is very large.

In this experiment we have to keep constant 1) the stimulation interval, 2) the intensity of the stimulating current and 3 ) the duration of the polarising current. They were 
1-5 min, $4 \mathrm{~m}$. A. and 10-30 sec. respectively according to the individualities of the cores. The intensity of the polarising current was varied from $10^{-1}$ to $10^{2} \mathrm{~m}$. A. The control was done in two ways, namely, by change of the resistance of the circuit and by change of the source potential. The results were identical.

The data obtained point out clearly that the anelectrotonus makes the wave length and the conduction velocity larger while the catelectrotonus makes them smaller, provided that the tonus is relatively strong. The phenomena were decidedly clear and definite under the polarising electrodes, where the wave length increased even to twice as long or decreased even to one-third. These are illustrated schematically in Fig. 5. The velocity could not be measured simultaneously, but it surely varied parallel with the wave length.

It is well known that in nerve the velocity becomes larger under the cathode and smaller under the anode. But as the pole, under which the excitation or the activation takes place, is opposite in nerve and model, the facts coincide with each other in that the velocity becomes larger under the pole where the excitation or the activation takes place.

A very strong catelectrotonus checks the conduction just as the strong anelectrotonus does in the nerve. Whether the "anodic depression", which corresponds to the "cathodic depression" in the nerve, can occur in the model or not, could not be made clear, because it was difficult to make a strong current slide into the core without activating it. If it is done, however, the wave will surely be stopped under or near the electrode, because the core will then be highly polarised anodically and the state will be very near that of the active iron, so that the activation potential, if evoked at all, will not be large enough to activate the neighbouring point.

As to the effects of the weak electrotonus, the examinations are not sufficient yet to say anything definitely, but as far as examined, they seem to be opposite to those of the strong tonus, that is, the anelectrotonus is inhibiting and the catelectrotonus is augumenting. This tendency was often noticed in cases of very weak polarisation in general, and also in the regions apart from the polarising electrode, where the tonus is more or less weak. In these regions, the wave length was often smaller than usual in the case of anodic polarisation and larger in the case of cathodic polarisation.

\section{Discussions}

The points of the results are

1. The so-called activation wave in Lillie's nerve model can be of certain limited lengths under some special conditions. Let it be called the "length" of the wave or simply the "wave length" for convenience sake. The most important condition for obtaining it is to stimulate (activate) the core in strong nitric acid, where the "repassivation" (recovery) can take place automatically after the activation. Now, this "length" can be large or small according to long or short immersion time and to large or small volume of the acid, the sheath fluid. In the case when the immersion time is. long enough, the length is comparatively large and is kept constant during the course of conduction. But if the immersion time is very short, the length, which is very small 
from the beginning, gets smaller and smaller while it is conducted (the decrement) until finally the conduction is stopped somewhere on the way.

2. The larger the length of the wave, the greater is the conductions velocity.

3. The strong anelectrotonus makes the wave length larger, while the strong catelectrotonus makes it smaller. A very strong catelectrotonus, probably also a very strong anelectrotonus, checks the conduction of the wave. The fact is that the activation wave goes on with increment or decrement of the wave length in the regions of an- or "catelectrotonus until it reaches the polarising electrode, and then, if not checked there, it proceeds on with decrement or increment to get back to the original size.

4. The weak anelectrotonus seems to be inhibiting, and the weak catelectrotonus augumenting.

The chief interest of the author is centred in the wave length itself and in the variations which it suffers in course of conduction.

Suppose, now, a certain change of the state and its restitution are taking place 'successively at a certain point in a medium, and this local event is being transmitted in , all directions in the medium, which is continuous and uniform. We have then, naturally, the progression of the change itself and the progression of the restitution process in succession. Consequently, the space, which will be occupied by the change itself at any given instant, will be limited. The activation wave of limited length in Lillie's model is nothing but a special example. The same can also be expected to occur in living nerve, namely, "the excitation wave of limitsd length". This length was once taken into consideration and was estimated to occupy a few centimetres. The estimation was made from the data obtained on the duration of the action potential and on its conduction velocity. The velocity, however, was thereby simply taken as invariable all along the nerve. Is it really so? Moreover, it was assumed that the "length" is nothing but an area left behind the active wave without any significance for further conduction. Is it correct?

In a previous paper on nerve conduction (Yamagiwa ${ }^{7}$, see also Appendix), the author discussed the problem on the length of the active area of nerve and on the variations which it will suffer in course of conduction. Upon this ground, he was able to explain satisfactorily the chief and essential facts concerning the nerve conduction. The fundamental idea was the "conduction from length to length" in place of the "conduction from point to point" as suggested in Hermann's core conductor theory. He did not mean, however, any discontinuous or jumping conduction from one length to another, because it is certain that the excitation in one length will begin at the part adjacent to the neighbouring active length, and, therefore, the conduction will appear as if it were a matter between two neighbouring points only. What he did claim was that there must always exist an active region of a certain length, which is capable of exciting another certain length, that is to say, not only the wave front but also the whole length of the wave is significant for the excitation of the next region. The essential questions will be, among others, 1) whether such a length actually appears or not, and 2) whether the length can be constant, decremental or incremental according to the circumstances, 
as is requested from the theory. These questions cannot be examined on the living nerve, nor on any other models than Lillie's, on which the questions were answered quite satisfactorily as was mentioned above.

Let us just suppose that a nerve fibre, a piece of wire, etc. is in a uniform state throughout its length, and that any change of the state and the recovery following that do go on with one and the same course of time at every point. What will happen, then, if the change at "one point" is the only cause for the change of the "next point", and if in this way the conduction goes on from "point to point"? It is certain, as discussed above, that the change will occupy a certain limited length at every given instant, but it is also certain that the length cannot but be constant, in other words, that the decrement or the increment cannot be the case during the course of conduction, because the activation wave is to proceed with a constant velocity and the recovery wave is to follow that with the same velocity after a certain definite time. In fact, however, it was clearly decremental when the immersion time was short, and it was clearly constant in the case of a long immersion time (the "increment" did not appear clearly in the present work. Perhaps it will, if we employ a proper method of stimulation, which enables us to activate the core exactly in any length desired). This fact suggests strongly that the mode of conduction, at least in Lillie's model, is not "from point to point" or "from unit to unit" in Hermann's sense.

The investigations of the influences of the electrotonus were planned from two motives. First, it was for examining the variation of the conducting wave length under those special conditions. In the method adopted, the grade of the tonus is higher near the polarising electrode and gradually weaker with distance. What will happen to the conducting wave length in such a condition? The result was that the wave behaves as a decremental or an incremental as it approaches the cathode or the anode, as theoretically expected. The second object was to survey the relation between the electrotonus and the conduction velocity as a special matter. The results agreed fairly well with the facts known in the nerve, namely, the augumenting effect of strong anelectrotonus (the catelectrotonus in physiology) and the inhibiting effect of strong catelectrotonus (the anelectrotonus in physiology) etc. These effects can be understood, though not exactly, it one thinks that the anodic current, the direction being the same with that of the activation current, will make the electric threshold for activation lower, while the cathodic current will make it higher, so that in the former case a relatively larger, and in the latter case a relatively smaller portion will be activated by a source of the same strength. In fact, the wave length was found larger under the anode and smaller under the cathode, as described before.

How will be, on the other hand, the activation potentials under the polarising electrodes? They were not measured, but it is to be smaller under the anode, and larger under the cathode, because the grade of passivity is decreased under the former and increased under the latter. Recognizing, then, the facts that under the cathode the wave length is small and the activation potential is large, while under the anode the wave length is large and the activation potential is small, we ought to conclude that the cathodic 
interruption must be due to the small wave length, while the anodic interruption, if occurred, to the small activation potential. In physiology, the cathodal block is usually interpreted as being due to the small action potential, and the anodal block as due to the small conduction velocity, which is said to fall even to zero. But the "block due to small conduction velocity" is difficult to understand. The author thinks that his conclusion, mentioned above, in which the "wave length" is taken up in place of the conduction velocity, is more reasonable and probable, because, according to his idea, the large or small wave length means the large or small stimulating power, and from this standpoint of view, the interruptions at the anode and the cathode can be both reduced to the same reason, that is, to the small stimulating power.

\section{SUMMARY}

Observations were made on the "length" of the activation wave in Lillie's nerve model. The results obtained can be summarised as follows:

1) The length of the wave depends on the grade of passivity, and the higher the latter, the larger the former, if other conditions remain unchanged. The waves are conducted with constant wave lengths, but in the case when the grade of passivity is too low, the wave length undergoes decrement until the conduction is interrupted somewhere on the way.

2) The wave length and the conduction velocity change parallel in magnitude.

3) The anelectrotonus makes the wave length larger, and the catelectrotonus smaller, provided that the tonus is strong. In extreme cases, the interruption of the conduction takes place under the cathode and possibly also under the anode. The effects of weak electrotonus seem to be opposite to those of the strong.

These facts bear each a special meaning of their own, but besides those, they can be, as a whole, a support of the author's theory on nerve conduction.

\section{LITERATURE}

1) Lillie, R. S.: J. gen. Physiol. 7, 473, 1925.

2) " : " 14, 349, 1931.

3) $\quad " \quad$ : Science 69, 305, 1929.

4) " : J. gen. Physiol. 3, 107, 1921.

5) $\quad$ : $\quad$ : 3, 129, 1921.

6) $"$ : " 13, 1, 1930.

7) Yamagiwa, K.: Nihon Seirigaku Zasshi (Jap. J. Physiol.) 5, 1, 1940.

\section{APPENDIX}

Abstract of "The action current as a stimulus" by K. Yamagiwa, Jap. J. Physiol., 5, 1, 1940.

\section{Part I.}

Be $x$ the portion of a fibre primarily excited, $y$ the portion to be excited by $x$, and $Y$ the point being excited by just the threshold (see Fig. 1). A non-medulated fibre is under consideration. (The medulated fibre, provided with Ranvier's nodes, can be taken as a special case). 
Let us assume

1) $i=I e^{-\eta y}$ (after Rashevsky)

$i$ : the action current across the membrane at the point $Y$.

$I$ : the same at the point $Y_{0}$.

$\eta$ : a constant in Rashevsky's equation.

2) $I=\int_{0}^{x} \frac{k V(t)}{W+R x} d x$

$V(t)$ : the action potential (a function of $t$ ).

$W:$ the membrane resistance at $Y_{0}$ or any other point.

$R$ : (Inner resistance+outer resistance) per unit length of the fibre.

3) $V(t)=k_{1}\left(e^{-\alpha t}-e^{-\beta t}\right), \alpha<\beta$

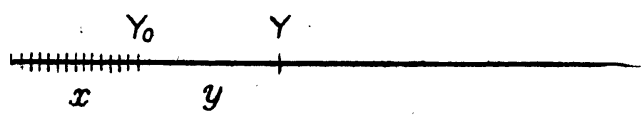

Fig. 1
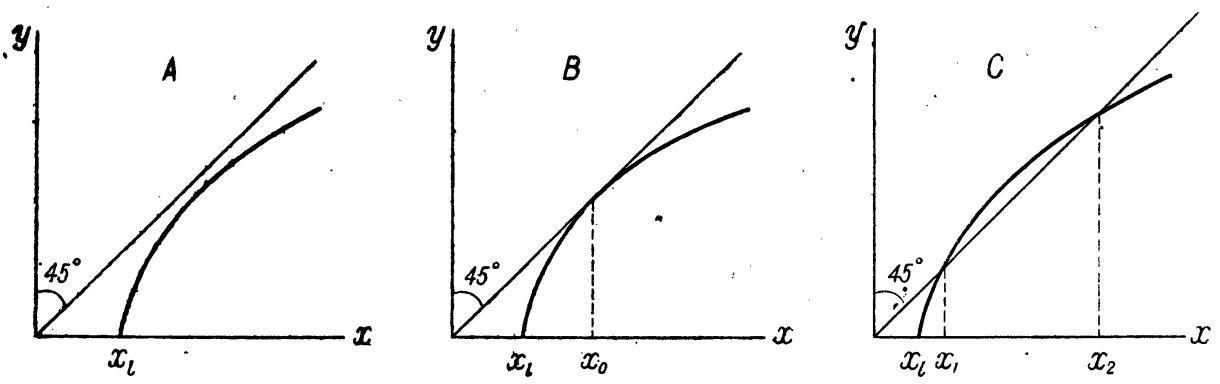

Fig. 2

Introducing (2) and (3) into (1), we get

$i=\frac{k k_{1}\left(e^{-\alpha t}-e^{-\beta t}\right)}{R} \cdot \log \left(1+\frac{R}{W} x\right) \cdot \mathrm{e}^{-\eta y}$

Let us adopt, as theoretical formulae for electric excitation, de Almeida's and Blair's ones, namely,

$d E=k_{2} i d t-2 k_{3} t E d t$ (de Almeida's first equation)

$d E=k_{2} i^{2} d t-2 k_{3} t E d t$ (de Almeida's second equatian)

$d E=k_{2} i d t-k_{3} E d t$ (Blair's equation)

Introduce (4) into (5), (6) and (7) and integrate. We get then

$$
\begin{aligned}
& E=\frac{k k_{1} k_{2}}{R} \log \left(1+\frac{R}{W} x\right) \cdot e^{-\eta y} \cdot e^{-k_{3} t^{2}} \int_{0}^{t} e^{k_{3} t^{2}}\left(e^{-\alpha t}-e^{-\beta t}\right) d t
\end{aligned}
$$

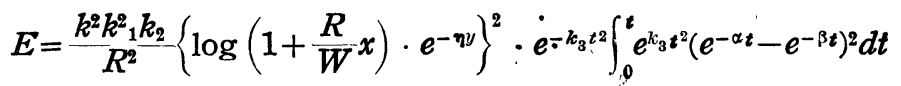

$$
\begin{aligned}
& E=\frac{k k_{1} k_{2} \log \left(1+\frac{R}{W} x\right) \cdot e^{-\eta y}}{R\left(k_{3}-\alpha\right)\left(k_{3}-\right)}\left[\left(k_{3}-\beta\right) e^{-\alpha t}+\left(\alpha-k_{3}\right) e^{-\beta t}+(\beta-\alpha) e^{-k_{3} t}\right]
\end{aligned}
$$

In either case, there is a maximum point in $E$ at a certain time, say $t_{m}$. Assuming 
as usual that the excitation takes place when $E$ attains a certain definite value, we put $E_{m a x}=c$, which leads us formally to the same result

$$
\log \left(1+\frac{R}{W} x\right) \cdot e^{-\eta y}=R K
$$

where $K$ is a constant involving $k, k_{1-3}, t_{m}, \alpha$ and $\beta$, and different in value in cach case.

It will perhaps be allowed, as a first approximation, to put $1 \gg \frac{R}{W} x$.

Then $\log \left(1+\frac{R}{W} x\right) \fallingdotseq \frac{R}{W} x$, hence from (11),

$$
\begin{aligned}
& \frac{R}{W} x \cdot e^{-\eta y}=R K \\
& \log \frac{R}{W} x-y y=\log R K \\
& y=\frac{1}{\eta}\left(\log \frac{R}{W} x-\log R K\right)=\frac{1}{\eta}\left(\log x+\log \frac{1}{W K}\right)
\end{aligned}
$$

According to the value of $\frac{1}{\eta} \log \frac{1}{W K}$, there can be three types of $x$-y-relation as illustrated in Fig. 2.

There are two important points to be noticed, namely, 1) if $x<x_{i}$, then $y<0$, in words, the conduction can never take place, unless $x$ is larger than a certain definite value, and 2) the type of $x-y$-relation can be different in different fibres, and also in different parts of one and the same fibre, for example, in normal and narcotised regions.

\section{Part II.}

The value of $y$ in the $x$-y-relation obtained represents the length which is to be excited by $x$ and $x$ only, but not the acutual length which will be excited by the moment $\boldsymbol{t}_{m}$. The actual length will be a little larger, because the part of $y$ adjacent to $x$ will be excited earlier and will give some effect upon the rest of $y$. If we call the actual length the "conducting length" (C.L.), the mean velocity of conduction should be given by $V=\frac{C . L .}{t_{m}}$. But the exact calculation of C.L. is a matter of etreme difficulty. Qualitative-analytically, however, the $x$-y-relation may be taken as approximately representing the $x$-C.L.-relation, in such a way that $x$ excites $y_{1}$, and $y_{1}$ excites $y_{2}$ etc. (Fig. 3 ), the same functional relation being kept in ( $x$ and $\left.y_{1}\right)$ and $\left(y_{1}\right.$ and $\left.y_{2}\right)$ and so on.

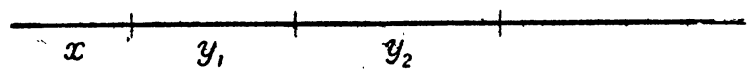

Fig. 3

We have then

in type A: $x<x_{l}$ : no conduction,

$x>x$ : conduction with decrement in C.L. (and $V$ ) till C.L. gets as small as $x$.

in type B: $x<x_{i}:$ no conduction,

$x<x_{0}:$ Decrement in C.L. $\rightarrow$ interruption on the way,

$x>x_{0}$ : Decrement in C.L. $\rightarrow$ constancy in C.L. $\left(x_{0}\right)$, 
in type $\mathbf{C}: \boldsymbol{x}<x_{l}:$ no conduction,

$x<x_{1}$ : decrement in C.L. $\rightarrow$ interruption on the way,

$x_{1}<x<x_{2}$ : increment in C.L. $\rightarrow$ constancy in C.L. $\left(x_{2}\right)$

$x>x_{2}$ : decrement in C.L. $\rightarrow$ constancy in C.L. $\left(x_{2}\right)$

Which type is the normal, cannot be decided for the moment, because we don't know the values of the physiological constants necessary for that. But if the type $C$ or $B$ be taken as the normal, we can understand most of the chief physiological facts known as to the conduction, fairly well:

1) The graded responses of the muscle fibre (To several lengths of $x$ given correspond several distances to be travelled in each type).

2) The decrement in the conduction velocity and the interruption of the wave in narcotised nerve (The type $\mathrm{C}$ and $\mathrm{B}$ change into the type $\mathrm{A}$ as the result of the changes of $W$ and $K$ in narcosis).

3) The parallelism between the fibre size and the conduction velocity (the difference in the fibre size can be taken as the difference in $\eta$ and $W$ ).

4) The relation between the temperature and the conduction velocity (the effects of temperature upon $\eta, W, K$ and $t_{m}$ were taken into consideration).

The expences for this work were defrayed by the National Research Council of Japan. 